\title{
Energy-Efficient Range Assignment in Heterogeneous Wireless Sensor Networks
}

\author{
Mihaela Cardei, Mohammad O. Pervaiz, and Ionut Cardei \\ Department of Computer Science and Engineering \\ Florida Atlantic University \\ Boca Raton, FL 33431, USA \\ E-mail: \{mihaela@cse., mpervaiz@, icardei@cse.\}fau.edu
}

\begin{abstract}
This paper considers a heterogeneous wireless sensor network consisting of a large number of energy constrained wireless sensor nodes and a connected subnetwork of resource-rich supernodes used for relaying sensor data to the user. We address the range assignment problem in this heterogeneous sensor network - selecting the transmission range for each sensor node such that a multihop communication path exists between each sensor node and a supernode. In order to balance power usage in the sensor network the objective of this range assignment problem is to minimize the maximum transmission power consumed at each sensor nodes.

This is the first paper to address this problem. We propose several solutions: an Integer Programming approach, a distributed greedy protocol, and a minimum spanning tree protocol based on clustering. We validate and evaluate the proposed solutions with simulations.
\end{abstract}

\section{INTRODUCTION}

Wireless sensor networks (WSNs) provide rapid, untethered access to information and computing, eliminating the barriers of distance, time, and location for many applications in national security, civilian search and rescue operations, surveillance, area/target monitoring, and many more.

In this paper we address topology control in heterogeneous WSNs consisting of two types of wireless devices: resource-constrained wireless sensor nodes deployed randomly in large numbers and a much smaller number of resource-rich supernodes, placed at known locations. The supernodes have two transceivers, one to connect to the sensor network, and another to connect to the supernode network. The supernode network provides better QoS and is used to quickly forward sensor data packets to the user. With this setting, data gathering in heterogeneous WSNs has two steps: first, sensor nodes transmit and relay measurements on multihop paths towards any supernode (see Figure 1). Once a data packet encounters a supernode, it is forwarded using fast supernode to supernode communication toward the user application. Additionally, supernodes could process sensor data before forwarding. Intel's study in [8] shows that using a heterogeneous

This work is supported in part by NSF grants CNS 0422762 and CCF 0545488 . architecture results in improved network performance, such as lower data gathering delay and longer network lifetime. Hardware components of the heterogeneous WSNs are now available commercially [3].

We model topology control as a range assignment problem for which the communication range for each sensor node must be computed. The objective is to minimize the maximum transmission power at all sensors, while maintaining a multihop communication path from all sensors to one supernode. The main difference compared with range assignment in ad hoc wireless networks is that here we are not concerned with connectivity between any two nodes. Our problem is specifically tailored to WSNs environment, in which data is forwarded from sensors to supernodes.

This is the first paper to study the range assignment problem in a heterogeneous WSN. The contributions of this paper are: (1) formulate the range assignment problem for heterogeneous WSNs (2) propose several algorithms for solving the HRA problem using integer programming, a greedy distributed protocol, and an MST cluster-based approach, (3) analyze the performance through simulations.

The rest of this paper is organized as follows. In section II we briefly present related works on heterogeneous WSNs and topology control problems. Section III describes the features of heterogeneous WSNs and introduces the HRA problem. We continue in section IV with our solutions for solving the HRA problem. In section $\mathrm{V}$ we present the simulation results, and section VI concludes our paper.

\section{RELATED WORK}

The benefits of using heterogeneous WSNs, containing devices with different capabilities, have been presented recently in literature. In [14], it is reported that properly deployed, heterogeneity can triple the average delivery rate and provide a 5 -fold increase in the network lifetime.

Topology control in ad hoc wireless networks has been addressed previously in literature in various settings. In general, the energy metric to be optimized (minimized) is the total energy consumption or the maximum energy consumption per node. Sometimes topology control is combined with other objectives, such as to increase the 
throughput or to meet some specific QoS requirements [9]. The strongly-connected topology problem with a minimum total energy consumption was first defined and proved to be NP-complete in [1], where an approximation algorithm with performance ratio of 2 for symmetric links is given. In general, topology control protocols can be classified as: (1) centralized and global vs. distributed and localized; and (2) deterministic vs. probabilistic. The localized algorithm is a special distributed algorithm, where the state of a particular node depends only on states of local neighborhood. That is, such a algorithm has no sequential propagation of states.

Most protocols are deterministic. The work in [13] is concerned with the problem of adjusting the node transmission power such that the resultant topology is connected or biconnected, while minimizing the maximum power usage per node. Two optimal, centralized algorithms, CONNECT and BICONN-AUGMENT, have been proposed for static networks. They are greedy algorithms, similar to Kruskal's minimum cost spanning tree algorithm. For ad hoc wireless networks, two distributed heuristics have been proposed, LINT and LILT. However, they do not guarantee network connectivity.

Among distributed and localized protocols, $\mathrm{Li}$ et al. [10] propose a cone-based algorithm for topology control. The goal is to minimize total energy consumption while preserving connectivity. Each node will transmit with the minimum power needed to reach some node in every cone with degree $\alpha$. They show that a cone degree $\alpha=5 \pi / 6$ will suffice to achieve connectivity.

$\mathrm{Li}$, Hou and Sha [11] devise another distributed and localized algorithm (LMST) for topology control starting from a minimum spanning tree. Each node builds its local MST independently based on location information of its 1-hop neighbors and only keeps 1-hop nodes within its local MST as neighbors in the final topology. The algorithm produces a connected topology with maximum node degree of 6 . An optional phase is provided where the topology is transformed to one with bi-directional links. An extension is given in [12], where the given network contains unidirectional links.

Our work differs from these approaches by considering the issue of providing a topological infrastructure specifically tailored for data gathering in heterogeneous WSNs. In such applications, the sensor to sensor connectivity is not relevant. Instead, it is necessary to provide data collection paths between sensors and supernodes.

\section{Problem Definition}

\section{A. Heterogeneous Network Architecture}

We consider a heterogeneous sensor network consisting of two types of wireless devices: resource-constrained wireless sensor nodes and resource-rich "supernodes", as illustrated in Figure 1.

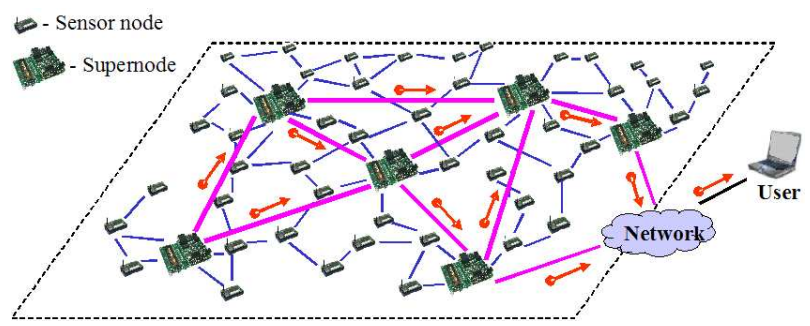

Fig. 1. Heterogeneous Wireless Sensor Networks.

Sensor nodes have low cost, limited battery power, short transmission range, low data rate (up to several hundred Kbps) and a low duty cycle. The main tasks performed by a sensor node are sensing, data processing, and data transmission/relaying. Supernodes have two radio transceivers, one for communicating with sensor nodes and the other for communicating with other supernodes. Supernodes are more expensive, have more power reserves, higher data rate, and better processing and storage capabilities than sensor nodes. The main task performed by a supernode is to relay data from sensor nodes to the user application.

\section{B. HRA Problem Definition}

Let us consider a heterogeneous WSN consisting of $N$ sensors $s_{1}, s_{2}, \ldots, s_{N}$ and $M$ supernodes $g_{1}, g_{2}, \ldots, g_{M}$, with $M \ll N$. The supernodes are pre-deployed in the sensing area, they are connected, and their main task is to relay data from sensor nodes to the user application. On the other hand, sensor nodes are deployed randomly in the area of interest. We assume that when each sensor is using a maximum transmission range, there exists a path from any sensor node to at least one supernode.

In this paper we consider that sensor nodes can adjust their communication ranges to one of $P$ predefined values $R_{1}, R_{2}, \ldots, R_{P}$, and $R_{\max }=R_{P}$. Our goal is to establish the transmission range of each sensor such that: 1) there is a communication path from each sensor to at least one supernode, and, 2) the maximum power consumed per sensor node is minimized. The first condition is needed to guarantee that data from every sensor reaches at least one supernode. The second condition is needed to ensure an energy-efficient design and balanced energy consumption for sensor nodes. Once a packet with data from a sensor reaches a supernode, it will be relayed to the user application using a separate, more capable and less resource-constrained supernode-only network.

One of the main differences between the topology control considered in this paper and topology control in ad hoc wireless networks is that here we are not concerned with assuring the connectivity between any two nodes (or any two sensors), but rather with providing a path from each sensor to one supernode. 
A sensor can communicate with another sensor or with a supernode if the Euclidean distance between nodes is less than or equal to the sensor's communication range. We consider the path loss communication model where the transmission power of a sensor $s_{i}$ is $p_{i}=r_{i}^{2}$ for a transmission range $r_{i}$.

The formal definition is given below:

\section{Definition 1: Heterogeneous WSNs Range Assignment (HRA) Problem}

Given an heterogeneous WSN with $M$ supernodes and $N$ energy-constrained sensors that can adjust their transmission ranges to one of the $P$ values $R_{1}, R_{2}, \ldots$, $R_{P}=R_{\max }$, determine the transmission range $r_{i}$ of each sensor $s_{i}$ such that (1) there exists a communication path from every sensor to a supernode, and (2) the maximum power consumed over all sensor nodes is minimized, i.e. $\max \left\{r_{1}^{2}, r_{2}^{2}, \ldots, r_{N}^{2}\right\}=$ minimum.

\section{SOLUTIONS FOR HRA PROBLEM}

In this section we propose three approaches for solving the HRA problem. First, we formulate the HRA problem as an Integer Programming using flows to emulate connectivity. The second solution is a distributed algorithm that uses a greedy approach, and the third solution is a cluster-based approach.

\section{A. Integer Programming Algorithm}

In this section we first formulate the HRA problem as an Integer Program (IP). Solving the IP is a centralized approach and assumes locations for sensors and supernodes are known.

In order to model the connectivity requirement, we model the heterogeneous WSN as a flow network $G=(V, E)$. The vertex set $V=$ $\left\{n_{1}, n_{2}, \ldots, n_{M}, n_{M+1}, \ldots, n_{M+N}\right\}$ contains the nodes in the heterogeneous WSN. The first $M$ nodes in $V$ are the supernodes and the last $N$ nodes are the sensor nodes. Please note that when we refer in general to a node $n_{i}$, this means that $n_{i}$ can be either a supernode or a sensor node. If we specify the index $i$ as being between $1 \ldots M$ then, we refer to a supernode. If $i>M$ then $n_{i}$ refers to a sensor node.

We define the neighborhood of a node $n_{i}$ as $\Gamma\left(n_{i}\right)=$ $\left\{n_{j} \in V \mid \operatorname{dist}\left(n_{i}, n_{j}\right) \leq R_{\max }\right\}$. Then we define the directed edge set $E=\left\{\left(n_{i}, n_{j}\right) \mid n_{i}, n_{j} \in V\right.$ and $n_{j} \in$ $\left.\Gamma\left(n_{i}\right)\right\}$.

Based on our assumption that when all sensors are using the maximum transmission range $R_{\max }$ there is a path from every sensor to at least one supernode, it follows that this condition is satisfied in the graph $G$ as well.

In order to model the connectivity of each sensor to a supernode, we use the flow concept, where flow enters the network through sensor nodes and is collected at the supernodes. Each sensor node is a source that inserts one unit of flow in the network. The supernodes act as sinks, and all of them together receive the $N$ units of flow.

\section{Given:}

- $M$ supernodes $n_{1}, n_{2}, \ldots, n_{M}$ and $N$ sensor nodes $n_{M+1}, \ldots, n_{M+N}$.

- the neighborhood of each node $n_{i}$ (sensor node or supernode) is computed as $\Gamma\left(n_{i}\right)=\left\{n_{j} \in\right.$ $\left.V \mid \operatorname{dist}\left(n_{i}, n_{j}\right) \leq R_{\max }\right\}$.

- a sensor can adjust its transmission range to one of the values $R_{1}, \ldots, R_{P}=R_{\max }$, and the corresponding transmission powers are $p_{1}, \ldots, p_{P}=p_{\max }$, where $p_{i}=R_{i}^{2}, i=1, \ldots, P$.

- $\operatorname{dist}\left(n_{i}, n_{j}\right)$ is the Euclidean distance between two nodes $n_{i}$ and $n_{j}$.

Integer Programming:

Objective:

Minimize $r^{*}$

Subject to the following constraints:

1)

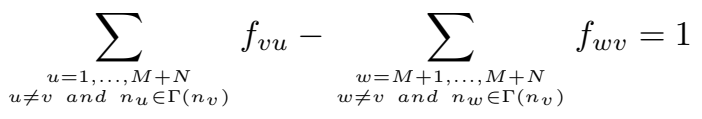

2)

$$
\sum_{j=1, \ldots, M} \sum_{\substack{v=M+1, \ldots, M+N \\ n \in \Gamma\left(n_{j}\right)}} f_{v j}=N
$$

3) $f_{u v} \leq x_{u v} N$ and $x_{u v} \leq f_{u v}$ for all $u=M+$ $1, \ldots, M+N, n_{v} \in \Gamma\left(n_{u}\right)$

4) $r^{*} \geq r_{u} \geq x_{u v} \cdot \operatorname{dist}\left(n_{u}, n_{v}\right)$ for all $u=M+$ $1, \ldots, M+N$, and $n_{v} \in \Gamma\left(n_{u}\right)$

5) $x_{u v}+x_{v u} \leq 1$, for all $u, v=M+1, \ldots, M+N$, and $n_{v} \in \Gamma\left(n_{u}\right)$

where:

- $x_{u v} \in\{0,1\}$ for $u=1, \ldots, M+N$ and $n_{v} \in \Gamma\left(n_{u}\right)$

- $f_{u v} \in\{0,1,2, \ldots, N\}$ if $u=M+1, \ldots, M+N$ and $n_{v} \in \Gamma\left(n_{u}\right)$, and $f_{u v}=0$ otherwise

- $r_{u} \in\left\{R_{1}, R_{2}, \ldots, R_{\max }\right\}$ for $u=M+1, M+$ $2, \ldots, M+N$

- $r^{*} \in\left\{R_{1}, R_{2}, \ldots, R_{\max }\right\}$

Boolean variables $x_{u v}$ indicate if there is a positive flow between neighboring nodes $n_{u}$ and $n_{v}$. We have $x_{u v}=1$ if there is a positive flow, and $x_{u v}=0$ if flow $f_{u v}=0$. Variables $f_{u v}$ represent the directed flow from node $n_{u}$ to node $n_{v}$. The flow function is defined only between neighboring nodes. Variables $r_{u}, u=M+1, \ldots, M+N$, represent sensor node transmission ranges. $r^{*}$ is the maximum range selected over all the sensors in the network. The total number of variables in our IP is upper-bounded 
by $2(M+N) \Delta+N+1$, where $\Delta$ is the maximum node degree in the graph $G$.

\section{Remarks on constraints:}

1) shows that sensor nodes are flow sources, and one unit of flow is inserted in each sensor node.

2) shows that supernodes act as sinks; in total $N$ units of flows will be received by all supernodes.

3 ) computes the boolean variables $x_{u v}$ based on the flow values.

4) computes the transmission range selected by each sensor node $n_{u}$ for $u=M+1, \ldots, M+N$. The set of positive out-flow values $\left(x_{u v}=1\right)$ gives the set of neighbors $n_{v}$ that have to be covered using the transmission range $r_{u}$. We also compute $r^{*}$, which is the maximum value between all sensor nodes transmission ranges.

5) prevents formation of flow loops between any neighbor nodes $n_{u}$ and $n_{v}$.

The solution of the IP problem returns the transmission range assigned to each sensor node, $r_{M+1}, \ldots, r_{M+N}$. The complexity of running this algorithm is dominated by the IP solver. In section $\mathrm{V}$ we present results for this IP problem computed with CPLEX [4].

\section{B. Greedy Algorithm}

In this section we propose a greedy algorithm that is run by each sensor node in order to decide its transmission range. We assume that if each sensor sets its transmission range to $R_{\max }$, the resulting sensor network topology would be connected, that is, there would be a path from each sensor to a supernode. Our goal is to minimize the node communication range while preserving connectivity to supernodes. We note with $p_{1}, p_{2}, \ldots, p_{\max }$ the power consumed by a sensor to transmit with communication ranges $R_{1}, R_{2}, \ldots, R_{\max }$.

In a global view, the algorithm grows trees rooted at supernodes. A sensor node gets attached to the tree that requires setting a minimum communication range. The algorithm starts with each supernode sending a Hello message with transmission power $p_{\max }$. Each sensor node begins the range selection protocol as soon as it receives a Hello message from a supernode or a BroadcastRA message from one of its neighbors.

A BroadcastRA message is sent by a sensor node that has decided its final communication range. This message announces the 1-hop neighbors about the selected range. We assume that a sensor is able to estimate the distance to a neighbor based on the RSSI of packets received from that neighbor.

Once a sensor receives a Hello or a BroadcastRA message, it is able to reach a supernode (possibly using multihop communication) by setting its communication range to the minimum value needed to reach that node. At this time, the sensor starts waiting, in eventuality that a message is received from a closer node, case in which a smaller communication range is needed. If the minimum range needed to reach the node from which the Hello or BroadcastRA message was received is $r_{i}$ then the waiting time $T_{i}$ is computed as $T_{i}=r_{i} \times \frac{W}{R_{\max }}$, where $W$ is the maximum waiting time. By using this formula, the sensor nodes with a larger $r_{i}$ will have to wait longer, increasing the probability that a BroadcastRA message from a closer node is received.

Once the waiting time expires, a sensor node $s_{i}$ sets its transmission range to the minimum value needed to reach the closest neighbor from which a BroadcastRA or a Hello message was received. Then $s_{i}$ broadcasts a message BroadcastRA $\left(s_{i}\right)$ announcing its 1-hop neighbors that it has set-up its final power level. BroadcastRA messages are always sent using the maximum power level $p_{\max }$.

Next, we present the pseudo-code for the power selection algorithm at a sensor node $s_{i}$

\section{Algorithm Greedy_HRA $\left(s_{i}\right)$}

1: /* assume that a BroadcastRA or Hello message is received from a node located at distance $d *$ /

2: find minimum value $r \in\left\{R_{1}, R_{2}, \ldots, R_{\max }\right\}$ such that $r \geq d$

3: $r_{\text {temp }} \leftarrow r$

4: start a timer $t \leftarrow r_{t e m p} \times \frac{W}{R_{\max }}$

5: while $t>0$ do

6: if BroadcastRA or Hello message is received from a node at distance $d^{\prime}$ and $d^{\prime}<r_{\text {temp }}$ then

7: find minimum value $r^{\prime} \in\left\{R_{1}, R_{2}, \ldots, R_{\max }\right\}$ such that $r^{\prime} \geq d^{\prime}$

8: $\quad / *$ update the waiting time and the range*/

9: $\quad t \leftarrow t-\left(r_{\text {temp }}-r^{\prime}\right) \times \frac{W}{R_{\max }}$

10: $\quad r_{\text {temp }} \leftarrow r^{\prime}$

11: end if

12: end while

13: sensor $s_{i}$ sets up its transmission range to $r_{\text {temp }}$ 14: send BroadcastRA $\left(s_{i}\right)$ message with power $p_{\max }$

If needed, we could also store a next-hop address pointing to the upstream node in the data gathering tree rooted in the nearest supernode. Figure 2 illustrates the data gathering network that results from running the above protocol in a random sensor network with 120 sensor nodes and a $3 \times 3$ supernode grid.

The complexity of this algorithm is $O(P \Delta)$, where $\Delta$ is the maximum node degree, that is the maximum number of nodes (sensor nodes or supernodes) located within distance $R_{\max }$ of a sensor.

\section{Clustering based Algorithm}

The distributed algorithm proposed in this section has two steps: (1) cluster formation, and (2) range assignment.

Let us describe first the cluster formation step. Each supernode serves as cluster head, and it broadcasts a 


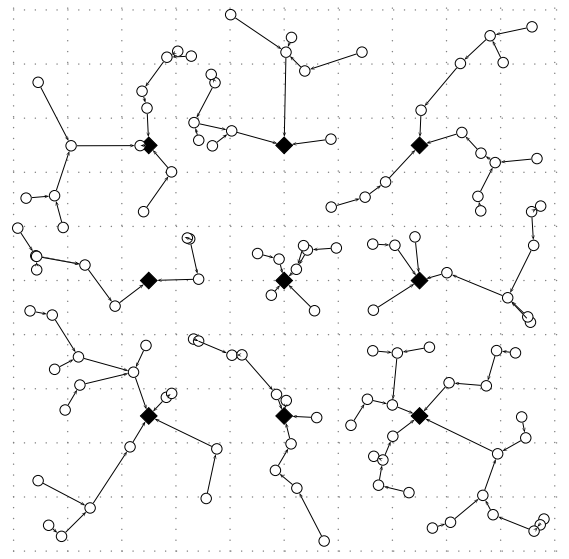

Fig. 2. Data gathering network for a random sensor network with 120 sensor nodes and a $3 \times 3$ supernode grid (diamonds).

CLUSTER_INIT (ID, hops) message containing the supernode id and the number of hops which is initially zero. Each sensor node maintains information about the closest supernode and forwards only messages from which it learns about a closer supernode:

1: min_hops $\leftarrow \infty$; cluster_id $\leftarrow$ NIL;

2: if CLUSTER_INIT(ID, hops) message received then

3: $\quad$ if cluster_id $\neq$ ID and hops $<$ min_hops then

4: $\quad$ cluster_id $\leftarrow$ ID

5: $\quad$ min_hops $\leftarrow$ hops

6: $\quad$ next_hop $\leftarrow$ sensor from which this message was received

7: rebroadcast the message CLUSTER_INIT(ID, hops +1 )

\section{8: $\quad$ end if}

9: end if

After a specific waiting time has passed, each sensor node joins the cluster cluster_id. Since we have $M$ supernodes, we will form $M$ clusters, with cluster-heads being $g_{1}, g_{2}$, $\ldots, g_{M}$.

In the range assignment step, the ranges are established such that there exists a path between any sensor $s_{i}$ and the cluster head of the cluster it belongs to.

Within each cluster, we proposed to use the local minimum spanning tree (LMST) algorithm [11] for setting up the communication ranges of each sensor nodes. LMST [11] is a localized algorithm, scalable, with good performances, and also provides strong connectivity within each cluster. Using this algorithm, each node sets-up its transmission range locally, using location information of its neighbors located within communication range $R_{\max }$. Each node uses this neighborhood to build a local MST, using Prim's algorithm. Then a node sets-up its transmission range to the minimum value needed to reach all one-hop neighbors in the local MST. It is proved in [11] that the resulting topology is strongly connected, that means a path exists between any two nodes. By applying LMST algorithm in each cluster, we will obtain a strongly connected topology for each cluster.

As an alternative, we could also use Gallager's distributed algorithm [5] for constructing a MST within each cluster. The algorithm [5] uses Kruskal's method [2] to build a MST and terminates in $5 n \log _{2} n$ time units, where $n$ is the number of participating nodes and a time unit is the message transmission time. Then each node sets its transmission range to the minimum range needed to reach its parent in the MST rooted at the cluster-head.

\section{Simulation Results}

In this section we present performance results for the algorithms introduced above: IP-HRA from section IV-A, Greedy-HRA from section IV-B, and Cluster-LMST from section IV-C.

We evaluated these algorithms with a custom packetlevel simulator implemented in C++. For IP-HRA we use the CPLEX [4] optimization library. The simulator assumes a reliable communication between neighbor nodes. We will address a non-ideal communication channel in our future work. Our measurements focused on the maximum communication range and on the network lifetime measured until the moment when the first mote depletes its initial energy reserve (1000 mAh).

The sensor network consists of Mica2 motes placed at random locations in a $200 \times 200 \mathrm{~m}$ area. The communication range can be varied between $10 \mathrm{~m}$ and $100 \mathrm{~m}$ in $2.5 \mathrm{~m}$ increments. Supernodes are deployed in a regular $p \times q$ grid, as in Figure 2.

The mote energy model is similar to the model used for LEACH [6] and accounts for both transmissions and receptions. The energy spent for transmissions is $E_{T x}(k, d)=$ $E_{\text {elec }} k+\epsilon_{a m p} k d^{2}$, where k is the packet length (bits), $\epsilon_{a m p}$ is a transmit amplifier parameter and $d$ is the distance. The receiver energy for a packet is $E_{R x}=E_{\text {elec }} k$. $E_{\text {elec }}=$ $50 \mathrm{~nJ} /$ bit is the energy dissipated per bit by the radio electronics for $\mathrm{Rx} / \mathrm{Tx}$.

Each sensor sends periodically a packet towards the supernodes. For data gathering, sensors route packets on the shortest hop count path towards a supernode. Intermediary motes perform data aggregation, reducing the number of forwarded packets. We used $0 \%$ and $50 \%$ aggregation factors. We run each simulation 10 times and we report the average values.

Figure 3 shows the maximum transmission range determined by the three algorithms in a network with 50 sensors. The number of supernodes changes from 1 to 15 arranged in grids from $1 \times 1$ to $5 \times 3$. As expected, IP-HRA produces the lowest maximum communication range, showing a slight decrease with the supernode count. Greedy-HRA starts higher but shows a significant decrease for the $2 \times 2$ supernode grid, followed by a gradual drop that 


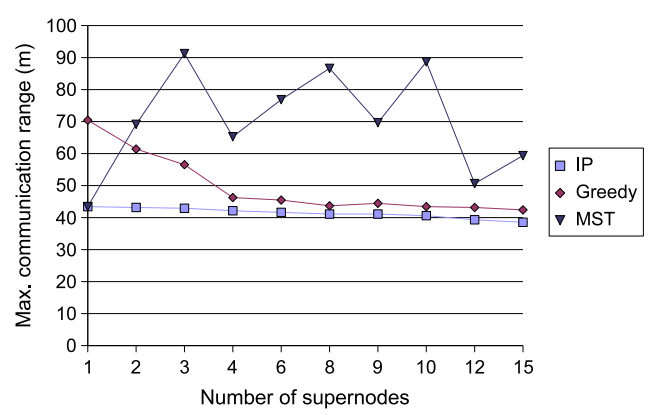

Fig. 3. Maximum communication range depending on supernode count.

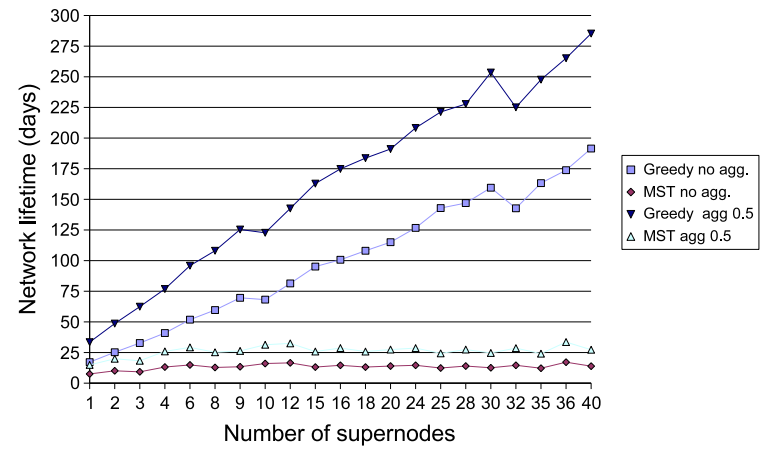

Fig. 4. Network lifetime depending on supernode count.

parallels $I P$-HRA's slow decrease. The MST-based protocol is more effective for a small number of supernodes $(1-3)$. Otherwise, Cluster-LMST's wide variation in the maximum communication range is due to the way the cluster and the MST are formed, sensitive to the local changes in supernode density. The hop-based MST approach permits sensors to select remote supernodes that may impact the maximum communication range.

The chart in Figure 4 shows the estimated network lifetime for the 200 mote network with various supernode populations and with/without data aggregation $(50 \%$ or 0\%). The Cluster-LMST protocol consistently features long paths with longer transmission distances, and thus does not benefit from a larger supernode population. This is due to the fact that in LMST, sensor nodes decide their transmission ranges locally, based only on the communication with the neighbors, and thus in most cases will not be largely affected by a uniform change in the number of supernodes. On the other hand, the greedy approach provides a more uniform and balanced load for motes located within transmission range to the supernodes (Figure 2). This allows an almost linear increase in network lifetime with the supernode population.

The simulation results can be summarized as follows:
- in WSNs with random sensor locations the network lifetime can achieve almost linear improvement with an increasing supernode population deployed in a uniform grid

- the Greedy-HRA protocol produces maximum communication ranges that come close to the optimal for supernode populations with uniform supernode densities.

\section{CONCLUSIONS}

In this paper we addressed the heterogeneous WSNs range assignment problem (HRA), used to adjust sensors' communication range in order to assure a communication path between every sensor and a supernode. Our main objective is to minimize the maximum transmission power over all sensors. We proposed several algorithms for computing sensor range assignments. An Integer Programming solution provides optimal ranges, albeit it is impractical in large scale WSN deployment. The distributed greedy protocol provides a scalable solution for range assignment that shows good scalability and linear improvement in network lifetime with supernode population. In our simulations we compared these two approaches with a cluster based minimum spanning tree approach.

For future work we plan to improve the greedy protocol for range assignment to deal with a lossy channel and to work with additional data gathering patterns, for both periodic and event-based data gathering.

\section{REFERENCES}

[1] W.-T. Chen and N.-F. Huang, The strongly connecting problem on multihop packet radio networks, IEEE Transactions on Communications, 37(3), pp 293-295, Mar. 1989.

[2] T. H. Cormen, C. E. Leiserson, R. L. Rivest, and C. Stein, Introduction to Algorithms, 2nd edition, McGraw Hill, 2001.

[3] Crossbow Mica2 motes and Stargate-Xscale, http://www.xbow.com

[4] CPLEX solver, http://www.cplex.com

[5] R. Gallager, P. Humblet and P. Spira, A Distributed Algorithm for Minimum-Weight Spanning Tree, ACM Transactions on Programming Languages and Systems, pp 66-77, 1983.

[6] W. R. Heinzelman, A. Chandrakasan, and H. Balakrishnan, EnergyEfficient Communication Protocol for Wireless Microsensor Networks, Proceedings of the 33rd Hawaii Intl. Conference on System Sciences, Jan. 2000.

[7] L. Hu, Topology Control for Multihop Packet radio Networks, IEEE Transactions on Communications, 41(10), 1993.

[8] Heterogeneous Networks with Intel XScale, http://www.intel.com/research/exploratory/heterogeneous.htm

[9] X. Jia, D. Li and D.-Z. Du, QoS Topology Control in Ad Hoc Wireless Networks, IEEE Infocom'04.

[10] L. Li, J. Y. Halpern, P. Bahl, Y.-M. Wang and R. Wattenhofer, Analysis of a Cone-Based Distributed Topology Control Algorithm for Wireless Multi-Hop Networks, Proc. ACM Symposium on Principles of Distributed Computing, pp 264-273, Aug. 2001.

[11] N. Li, J. Hou and L. Sha, Design and Analysis of an MST-based Topology Control Algorithm, IEEE Infocom'03.

[12] N. Li and J. Hou, Topology Control in Heterogeneous Wireless Networks: Problems and Solutions, IEEE Infocom'04.

[13] R. Ramanathan and R. Rosales-Hain, Topology Control of Multihop Wireless Networks Using Transmit Power Adjustment, IEEE Infocom 2000, pp 404-413.

[14] M. Yarvis et al., Exploiting Heterogeneity in Sensor Networks, IEEE INFOCOM, 2005. 